\title{
A Case of a Huge Inferior Vena Cava Leiomyosarcoma: Precise Preoperative Evaluation with Gadobutrol-Enhanced MRI
}

This article was published in the following Dove Press journal: Cancer Management and Research

\author{
Xiaoqi Zhou',* \\ Meng Wang ${ }^{1} * *$ \\ Shaoqiang $\mathrm{Li}^{2}$ \\ Huasong Cai ${ }^{1}$ \\ Lijian Liang ${ }^{2}$ \\ Zi-Ping $\mathrm{Li}^{1}$ \\ Shi-Ting Feng ${ }^{\prime}$ \\ Zhenpeng Peng' \\ Xuehua $\mathrm{Li}^{\prime}$
}

'Department of Radiology, The First Affiliated Hospital, Sun Yat-Sen University, Guangzhou 510080,

Guangdong, People's Republic of China;

${ }^{2}$ Department of Hepatic Surgery, The

First Affiliated Hospital, Sun Yat-Sen

University, Guangzhou 5I0080,

Guangdong, People's Republic of China

*These authors contributed equally to this work
Correspondence: Zhenpeng Peng;

Xuehua Li

Email 37595198@qq.com;

lixuehua803@163.com
Background: Leiomyosarcoma of the inferior vena cava (IVC) is a rare malignant tumour with poor prognosis. Surgical resection is the first line of treatment to achieve the best possible outcome. However, precise preoperative evaluation is essential to guide therapeutic decisions. Here, the preoperative evaluation potential of gadobutrol-enhanced magnetic resonance imaging (MRI) was assessed in the management of a 42-year-old patient with a large IVC mass.

Methods: The patient first underwent enhanced computed tomography (CT), but the relationship between the left renal vein and the mass in the dilated IVC was ambiguous, and it remained unclear whether the right hepatic vein was invaded by the lesion. To make a precise assessment of the tumour, the patient subsequently underwent high-resolution MRI angiography examination combined with high-concentration contrast medium gadobutrol.

Results: MRI demonstrated the integrity of the right hepatic vein and the left renal vein. Following a multidisciplinary consultation, a complicated surgery including complete resection of the mass, artificial vessel replacement of IVC, total hepatectomy, and bilateral nephrectomy with liver and kidney auto-transplantation was performed successfully. The surgical plan formulated after reviewing the MRI preoperatively was adhered to the course of the surgery. Postoperative CT re-examination showed that the blood flow of the artificial blood vessel and the right hepatic vein was unobstructed. Histopathological examination confirmed the tumour to be a leiomyosarcoma.

Conclusion: Preoperative imaging diagnosis and assessment have important implications for the surgical planning of IVC leiomyosarcoma. High-resolution MRI angiography examination with high concentration contrast medium gadobutrol may be of particular benefit in IVC tumours.

Keywords: leiomyosarcoma, inferior vena cava, preoperative care, magnetic resonance imaging, gadobutrol

\section{Introduction}

Leiomyosarcoma (LMS) is an aggressive soft tissue sarcoma derived from smooth muscle cells typically of uterine, gastrointestinal, or soft tissue origin. Approximately $2 \%$ of LMS originate from vessels, half of which occur in the inferior vena cava (IVC). About $50 \%$ of venous sarcomas are IVC LMS, accounting for $0.5 \%$ of all operated soft tissue LMS in adults. ${ }^{1}$ These tumours grow slowly both intra- and extraluminally, with invasion into adjacent structures and infrequent metastasis. $^{2}$ IVC LMS are classified into three groups according to the IVC segment involved: lower segment, or level 1 (infrarenal IVC); middle segment, or level 
2 (IVC between the renal and the hepatic veins); and upper segment, or level 3 (IVC between the hepatic veins and the right atrium). According to previous reports, the average length of IVC LMS is $12 \mathrm{~cm}^{3}$ The most frequently involved segment is the middle segment, accounting for $43 \%$ to $83 \%$ of IVC LMS. ${ }^{3-5}$ Clinical symptoms depend on the location, growth pattern, and segment involvement of the tumour. They include oedema of the lower extremities, renal and hepatic failure when there is invasion of the corresponding veins, and cardiac symptoms when IVC LMS extends to the right atrium. ${ }^{6,7}$ The tumour usually involves more than one segment of the IVC by the time it becomes clinically obvious, resulting in a combination of non-specific but life-threatening signs and symptoms ${ }^{8}$ leading to high mortality. Therefore, appropriate and preemptive treatment is essential for prolonged survival and better prognosis. Regardless, to date, no consensus exists on the management of IVC LMS, and complete surgical resection is regarded as the first-line therapy. ${ }^{9-11}$ Preoperative imaging plays an important role in clinical management as it allows not only accurate preoperative diagnosis but also the evaluation of the tumour surgery scope, margin, and anatomical relationship of the tumour to adjacent organs and structures. For this purpose, computed tomography (CT) and magnetic resonance imaging (MRI) are the main non-invasive methods used. ${ }^{12}$

Since first reported in 1871, many studies have described the imaging characteristics of IVC LMS. On CT scan, the tumour usually appears as a lobulate, irregular, heterogeneous, and hypoattenuated mass. On MR images, the tumour shows a long $\mathrm{T} 1$ and long T2 signal intensity with strong heterogeneous enhancement. The attenuation, signal intensity, and enhancement pattern mainly depend on the cystic components of the lesion. Although the other imaging findings vary depending on the tumour location, the most important sign for diagnosis is the dilation of the IVC lumen. Extensive collateral circulation is also detected around the lesion due to slow tumour growth.

One clinical challenge is to precisely describe the tumour extent and anatomical relationship with surrounding structures. On CT images, tumour obstruction and intravascular eddy currents hamper the filling of the IVC with contrast agent, making the observation of vascular lesions and clinical assessment difficult. Nevertheless, many case reports have used MRI for IVC LMS diagnosis. ${ }^{8,12-18}$ Compared with CT, MRI can provide more accurate information about the tumour's location, extent, collateral vessels, and adjacent structures with superior soft-tissue resolution, special sequences, and safer contrast material. The T2-weighted black blood sequence and high concentration contrast agent can overcome the limitations of $\mathrm{CT}$, clearly revealing the vessel walls and lumens of IVC and collateral vessels. More specifically, gadobutrol, a high concentration extracellular non-ionic macrocyclic gadolinium-based contrast agent, provides significantly higher mean signal-to-noise ratio values in large vessels in the abdomen than that of conventional gadolinium contrast medium and visualises the small vessels better. ${ }^{19}$ To our knowledge, no previous studies have used this high-concentration contrast medium in the diagnosis and preoperative evaluation of IVC tumours.

Herein, we report a case of a very large IVC LMS mass in which we compared CT and MR images, and demonstrated the important role of MRI for diagnosis and evaluation of the mass. The patient provided written informed consent for the case details and accompanying images to be published.

\section{Patient History}

A 42-year-old male patient presented to our hospital with complaints of abdominal discomfort, mainly in the upper quadrants, which persisted for 4 months, and bilateral symmetrical lower limb oedema for half a month. No other symptoms were reported, and physical examination was normal. Laboratory studies revealed the following blood concentration levels: creatinine, $207 \mu \mathrm{mol} / \mathrm{L}$ (reference range: 53-115 $\mu \mathrm{mol} / \mathrm{L}$ ); uric acid, $684 \mu \mathrm{mol} / \mathrm{L}$ (reference range; $200-430 \mu \mathrm{mol} /$ $\mathrm{L}$ ); alanine aminotransferase, $60 \mathrm{U} / \mathrm{L}$ (reference range; $<40 \mathrm{U} / \mathrm{L}$ ); serum lactate dehydrogenase, $271 \mathrm{U} / \mathrm{L}$ (reference range: $<240 \mathrm{U} / \mathrm{L}$ ); CA-125, $81.1 \mathrm{U} / \mathrm{mL}$ (reference range: $<35 \mathrm{U} / \mathrm{mL}$ ); and squamous carcinoma antigen, $1.7 \mu \mathrm{g} / \mathrm{L}$ (reference range: $<1.5 \mu \mathrm{g} / \mathrm{L}$ ). Ultrasonography from the local hospital suggested a space-occupying lesion in the IVC.

\section{Imaging Findings}

The patient initially underwent a computed tomography angiography (CTA) examination on a dual-energy MDCT scanner (Somatom Force, Siemens Medical Solutions, Forchheim, Germany), including the unenhanced phase, arterial phase, and venous phase, with a slice thickness of $0.5 \mathrm{~mm}$. A non-ionic contrast agent (iopromide, Ultravist 370, Bayer Schering Pharma, Germany) was 

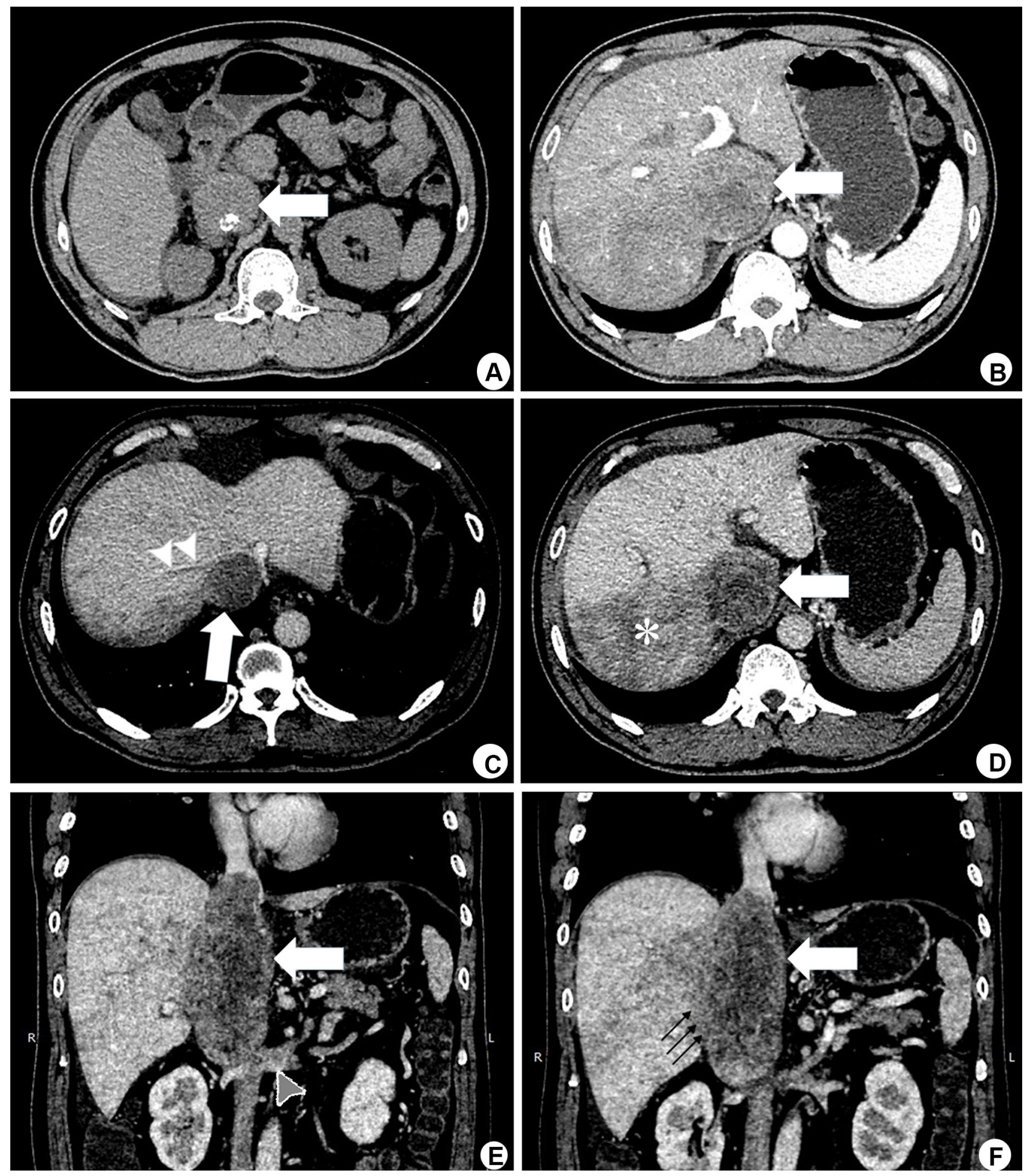

Figure I Preoperative CT images of intraluminal leiomyosarcoma of IVC. (A) Axial unenhanced CT image shows a slightly hypoattenuated mass (white arrow) in the dilated inferior vena cava with nodular calcification. (B) Axial contrast-enhanced arterial phase CT image shows dilation of the inferior vena cava. The lumen is filled with a mass showing mild heterogeneous enhancement (white arrow). The boundary between IVC and the right liver lobe is ambiguous. (C and $\mathbf{D})$ Axial contrast-enhanced venous phase CT image shows no significant enhancement in the centre of the mass (white arrow). The right posterior liver lobe shows heterogeneously inferior enhancement (star area), with right hepatic vein ill defined (white arrowheads). (E) Reconstructed coronal contrast-enhanced venous phase CT image shows the indistinct boundary between the tumour (white arrow) and the left renal vein (grey arrowhead with white outline). (F) Reconstructed coronal contrast-enhanced venous phase CT image shows the unclear (white arrow) boundary between right liver lobe and the hepatic segment of IVC (small black arrows). 


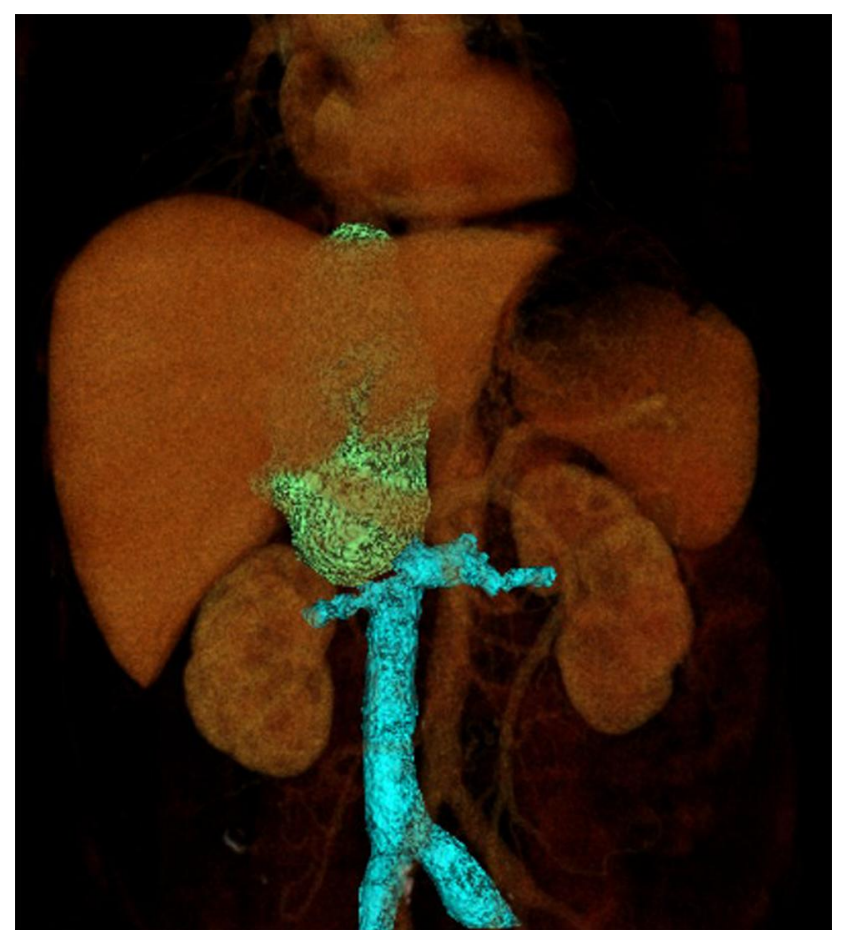

Figure 2 CT 3D reconstruction shows the poorly filled IVC and bilateral renal vein coloured in blue, with the LMS coloured in green.

injected into the antecubital vein at a rate of $3.5 \mathrm{~mL} / \mathrm{s}$ with a power injector of $1.5 \mathrm{~mL} / \mathrm{kg}$. Scan delay for the arterial phase and the venous phase was $34-37 \mathrm{~s}$ and $60-70 \mathrm{~s}$, respectively.

CT images revealed the lesion to be a heterogeneous soft-tissue mass within the dilated IVC hepatic segment. The lesion extended from the entrance of the right atrium to the level of the right renal vein, with a maximum diameter of $55 \mathrm{~mm}$ and a length of $139 \mathrm{~mm}$. In the arterial phase of the contrast-enhanced CT images, the mass showed mild, heterogeneous enhancement, with numerous tumour vessels. The venous phase showed no enhancement in the centre of the mass. A portion of the edge between the mass and the liver was unclear. Simultaneously, the right posterior liver lobe revealed heterogeneous low enhancement, without filling of the right hepatic vein. The left renal vein was pushed down and was not clearly demarcated from the mass (Figures 1 and 2).

However, evaluation of the involvement of the right hepatic vein and the left renal vein is crucial, as it largely guides the decision on which therapeutic regimen to follow. Had the tumour grown along the right hepatic vein, an additional right posterior hepatic lobectomy would be necessary. Had the left renal vein been invaded, the left kidney may have required excision. Radical surgery, accurate evaluation of the tumour and the scope of surgery is essential.

MRI examinations were thus performed using a 3.0-T system (GE SIGNA Pioneer, GE Medical Systems, Milwaukee, WI, USA) with unenhanced imaging (in-phase and opposed phase T1-weighted images; T2weighted images; high-resolution T2-weighted black blood sequence with and without fat-suppression; and diffusionweighted images) and contrast-enhanced T1-weighted imaging. The specific parameters for each sequence are

Table I Magnetic Resonance Imaging Protocol

\begin{tabular}{|c|c|c|c|c|c|c|c|c|}
\hline Parameters & $\begin{array}{l}\text { TI- } \\
\text { Weighted } \\
\text { in and } \\
\text { Opposed } \\
\text { Phases }\end{array}$ & $\begin{array}{l}\text { T2- } \\
\text { Weighted } \\
\text { Images }\end{array}$ & $\begin{array}{l}\text { High-Resolution } \\
\text { T2-Weighted } \\
\text { Black Blood } \\
\text { Sequence }\end{array}$ & $\begin{array}{l}\text { Fat-Suppressed High- } \\
\text { Resolution T2- } \\
\text { Weighted Black Blood } \\
\text { Sequence }\end{array}$ & Focus & $\begin{array}{l}\text { Diffusion- } \\
\text { Weighted } \\
\text { Imaging* }\end{array}$ & DISCO & SSFSE \\
\hline Repetition time (msec) & 5.5 & 10,588 & II,250 & 6316 & 8677 & 5455 & 5.7 & 900 \\
\hline Echo time (msec) & 1.8 & 85.8 & 85.9 & 85.9 & 64.2 & 77.2 & 2.1 & 70.8 \\
\hline Field of view $(\mathrm{cm})$ & 34 & 34 & 32 & 32 & 24 & 36 & 38 & 44 \\
\hline Scan matrix & $320 \times 288$ & $320 \times 320$ & $320 \times 320$ & $320 \times 320$ & $160 \times 64$ & $128 \times 128$ & $288 \times 256$ & $384 \times 256$ \\
\hline Slice thickness (mm) & 3 & 6 & 3 & 3 & 3 & 6 & 3 & 8 \\
\hline Slice gap $(\mathrm{mm})$ & -1.5 & 1.0 & 0 & 0 & 0 & 0.6 & -1.5 & 10 \\
\hline Motion compensation & - & - & Propeller & Propeller & - & - & - & - \\
\hline Flip angle $\left({ }^{\circ}\right)$ & 12 & 160 & $\mathrm{III}$ & 111 & 90 & 90 & 12 & 180 \\
\hline Number of slices & 66 & 26 & 30 & 30 & 30 & 36 & 70 & 9 \\
\hline Number of excitations & 1 & 1 & 4 & 4 & $2 / 16$ & $2 / 4$ & I & I \\
\hline Fat saturation & Yes & No & No & Yes & Yes & Yes & Yes & No \\
\hline
\end{tabular}

Note: *B factors, 50 and $800 \mathrm{~s} / \mathrm{mm}^{2}$.

Abbreviations: DISCO, Differential Subsampling with Cartesian Ordering; SSFSE, single shot fast spin echo. 

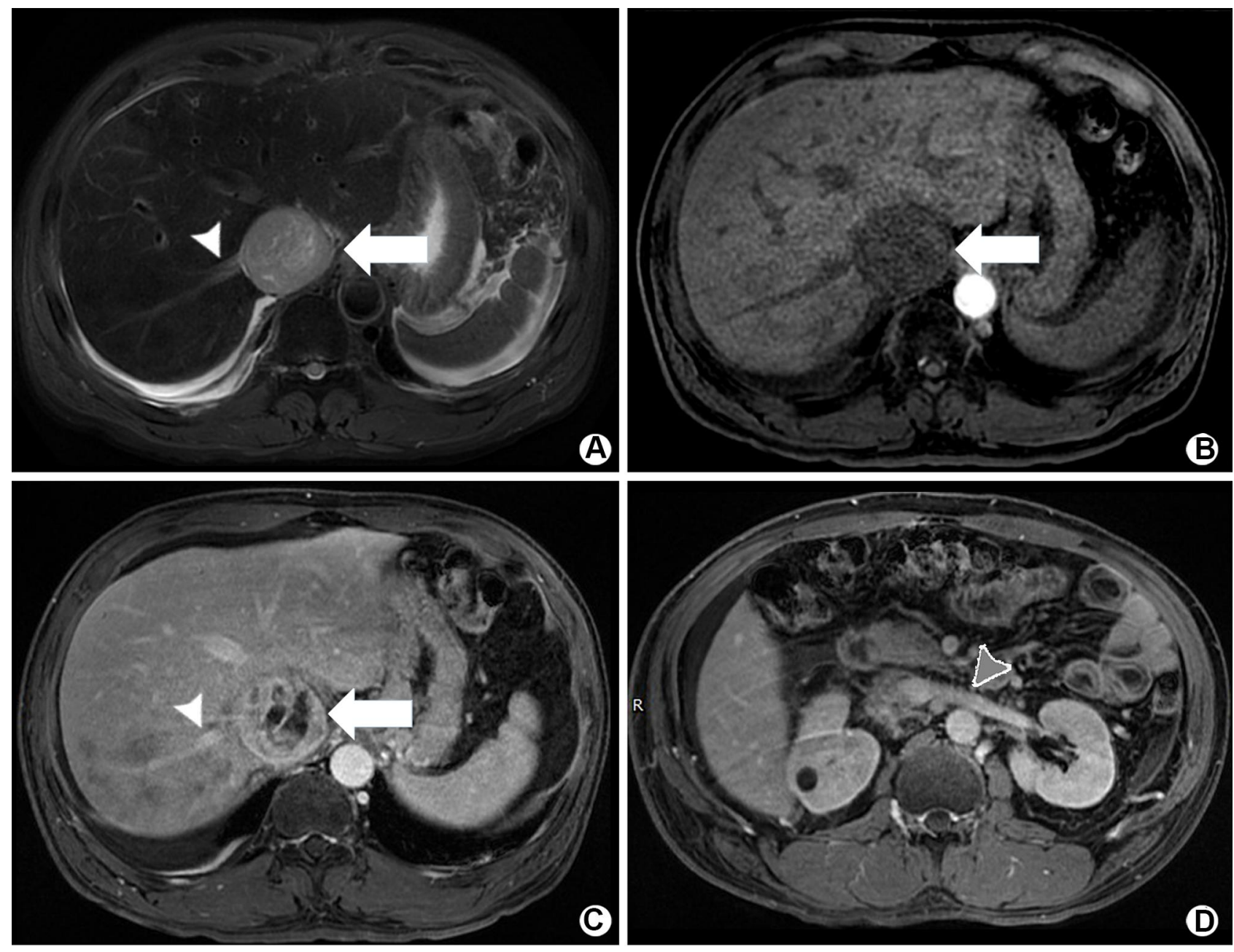

Figure 3 Preoperative axial T2-weighted black blood sequence with fat-suppressed MR image. (A) and TI-weighted with fat-suppressed MR image (B) shows the mass within the inferior vena cava with smooth vessel wall of IVC (white arrow). (C) Axial gadobutrol-enhanced late venous phase TI-weighted MR image shows heterogeneous enhancement of the mass (white arrow). The right hepatic vein is relatively hyperintense on T2WI (A) and enhanced TIWI images (C) (white arrowhead), without any abnormal signal intensity filling-defect. (D) Axial gadobutrol-enhanced TI-weighted fat-suppressed MR image shows the integrity of left renal vein, without any filling-defect (grey arrowhead).

summarised in Table 1. Moreover, $0.1 \mathrm{mmol} / \mathrm{kg}$ of nonionic gadolinium-based contrast medium (Gadobutrol, Gadovist, Bayer Schering Pharma, Germany) was injected at a rate of $1.5 \mathrm{~mL} / \mathrm{sec}$ utilising a power injector followed by a $30 \mathrm{~mL}$ saline flush.

Fat-suppressed T2-weighted images showed large hyperintense areas within the lesion. Axial T1-weighted gradient echo in- and out-of-phase MRI images showed the absence of fat-containing areas within the lesion. The lesion was homogeneously hypo-intense on T1-weighted images and heterogeneously enhanced after contrast agent injection on enhanced T1-weighted fat-suppressed MRI images. The right posterior liver lobe showed the same enhancement pattern as that on CT images, without intrahepatic metastatic lesions. The right hepatic vein revealed delayed enhancement and its orifice was completely blocked by the IVC tumour. Dilated accessory right hepatic veins were observed, indicating congestion of the right posterior liver lobe, with heterogeneous density decrease and uneven perfusion. The thin layer T2-weighted black blood sequence revealed the integrity of the vessel walls of the left renal vein and the right hepatic vein (Figures 3-5). On the diffusion-weighted image, the tumour showed hyperintensity, with the corresponding apparent diffusion coefficient map showing areas of decreased coefficient within the lesion, suggesting a limited diffusion of water molecules in the tumour (Figure 6).

After a multidisciplinary consultation, the patient underwent an extremely complicated operation of nine hours duration. The right hepatic vein and the renal vein were confirmed to be unaffected by the tumour and were spared. 

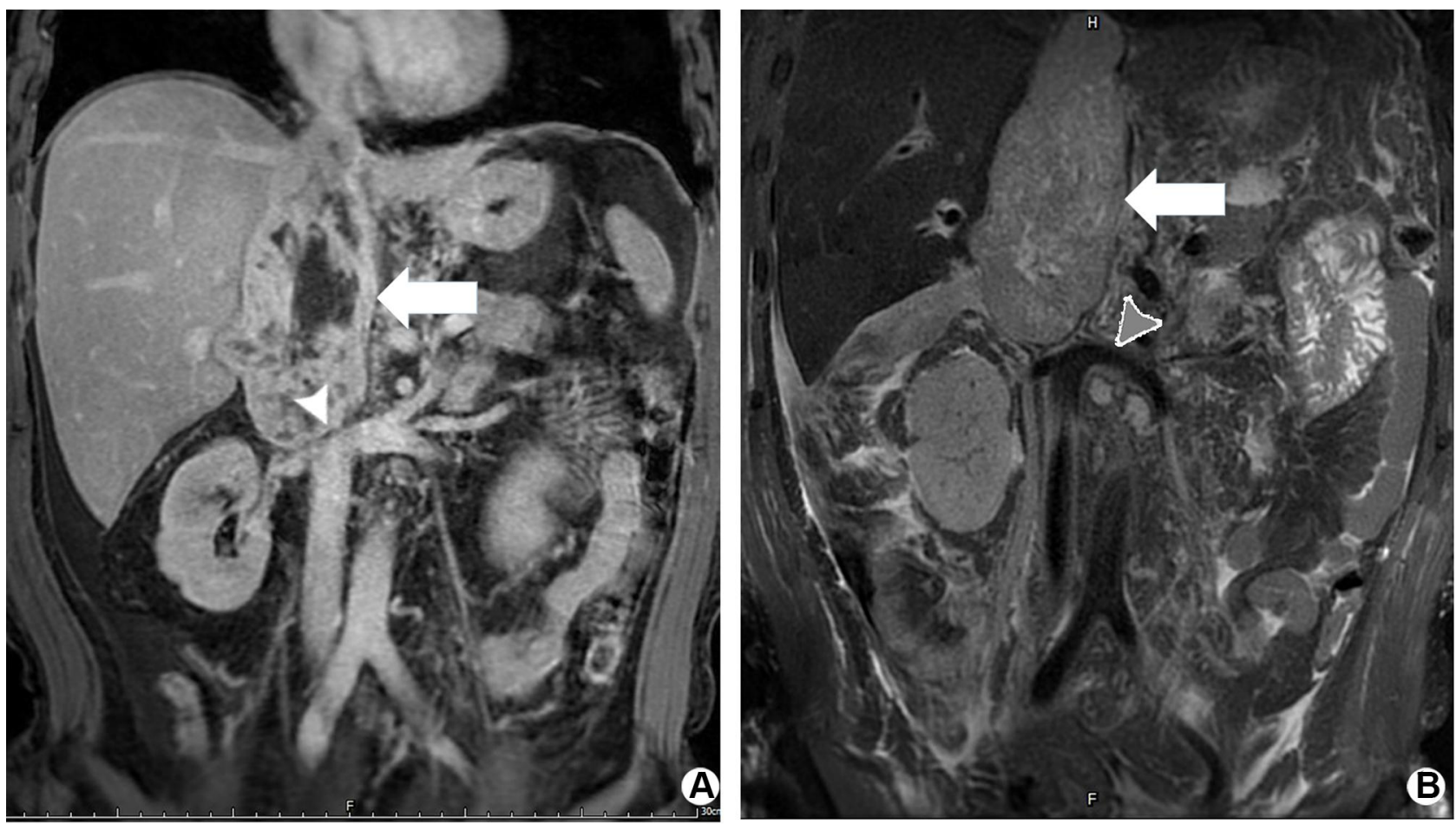

Figure 4 Preoperative coronal enhanced TIWI images. (A) and T2-weighted black blood sequence with fat-suppressed images (B) shows the mass within IVC (white arrow) and the relationship between the mass and bilateral renal veins (arrow heads). The mass and the left renal vein are totally separated. Enhanced TIWI image proves the patency of bilateral renal veins, without any filling defect (white arrowhead). T2-weighted black blood sequence shows complete vessel wall of renal veins (grey arrowhead).

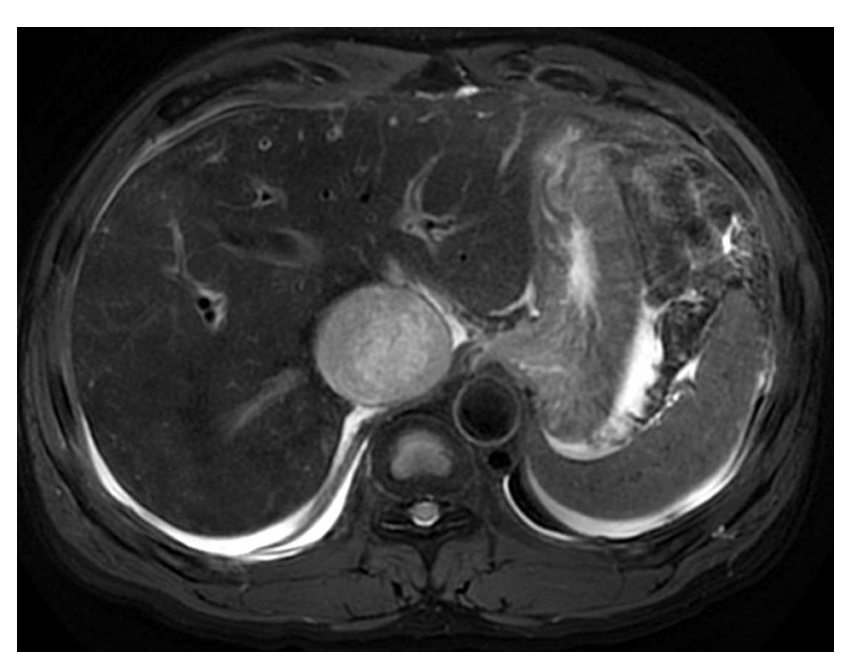

Figure 5 Liver parenchyma with oedema and congestion shows patchy hyperintensity on fat-suppressed T2-weighted axial MR image.

The liver and the kidney were resected and prepared for an autograft. The segment of IVC extending from the entrance of the right atrial to the left renal vein was resected and replaced by an artificial vessel. Finally, the right renal vein and the hepatic veins were anastomosed to the new IVC, and perfusion was restored. The scope of surgery was the same as that revealed on MRI, confirming that MRI is able to provide more precise evaluation than $\mathrm{CT}$ (Figure 7). Postoperative histological and immunohistochemical analyses confirmed an IVC leiomyosarcoma (Figure 8). The tumor was composed of spindle cells arranged in a slightly braided pattern with abundant cytoplasm, large and deep staining of nuclei, obvious heterogeneity, neoplastic necrosis, and pathological karyokinesis. The immunohistochemical results of the tumor cells were: H-Caldesmon (+), Actin $(+)$, S-100 (-), EMA (-), CD34 (-), Myogenin (-), MyoD1 $(-)$, CK $(-)$, INI-1 (+).

Following surgery and before being returned to the ward for close monitoring, the patient was quickly reexamined through CTA using the same MDCT scanner and contrast material as the previous time. The scan delay for the arterial phase, venous phase, and delayed phase was $34-37 \mathrm{~s}, 60 \mathrm{~s}$, and $180 \mathrm{~s}$, respectively. The arterial phase revealed a well-filled and clear artificial IVC segment with normal flow. The hepatic and renal veins were preserved and ensured a normal flow to the artificial IVC. Local haemorrhage was detected, but without active bleeding (Figures 9 and 10). The patient recovered well and was discharged after about two weeks of observation. 

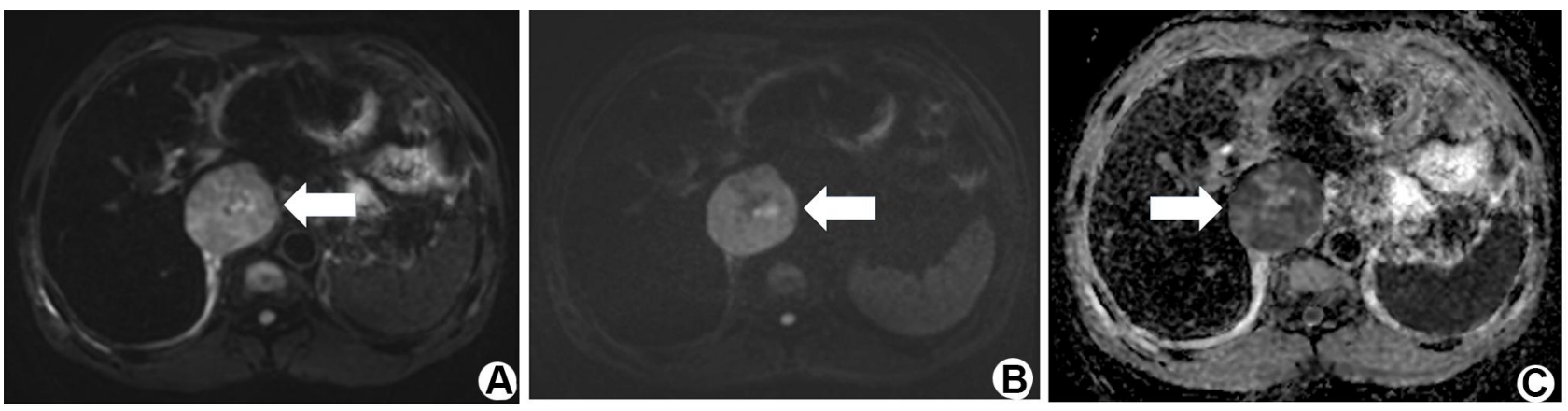

Figure 6 Preoperative diffusion-weighted images with $b=50(\mathbf{A})$ and $b=800(\mathbf{B})$ both show hyperintensity of the IVC mass (white arrow). (C) The tumour (white arrow) displays decreased apparent diffusion coefficients.

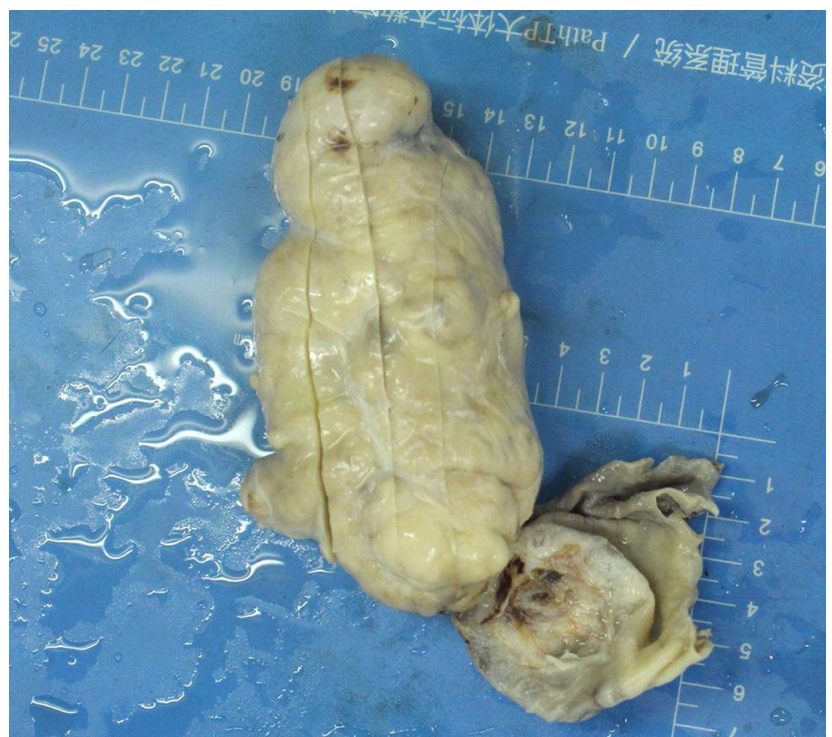

Figure 7 Gross pathology of the resected specimen from the patient.

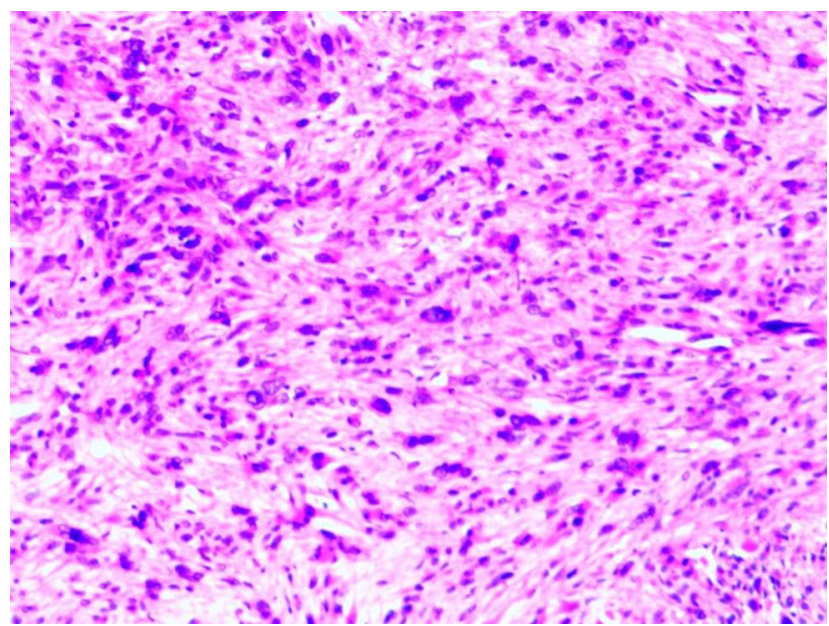

Figure 8 Photomicrograph of the tumour shows proliferating spindle-shaped cells with nuclear atypia in a braided arrangement. (HE stain, original magnification $\times 200$ ).

\section{Discussion}

Here, we report a clinical case of a bulky LMS originating from the IVC involving the right atrium orifice and the junction of hepatic veins and bilateral renal veins, for which combined liver and kidney autotransplantation was performed following tumour resection. This is the second reported case of IVC tumour resection combined with multiple organ autotransplantation worldwide since $2015 .^{20}$ High concentration contrast medium gadobutrol-enhanced MRI with high-resolution T2-weighted black blood sequence showed great accuracy in preoperative diagnosis and tumour extent identification. The findings helped guide a complex, radical surgical operation involving multiple organs that led to the survival of the patient.

Several studies have reported that CT can provide sufficient evidence for accurate diagnosis and tumour extent determination of IVC LMS. ${ }^{8,12,13}$ Contrastenhanced $\mathrm{CT}$ is considered a sensitive tool for the diagnosis and follow-up of IVC leiomyosarcoma as it delineates the intravascular component of the tumour. However, CTA has several limitations. While it is advantageous for arterial diseases, its diagnostic performance for diseases affecting veins and small vessels remains unsatisfactory. CT also has inferior soft tissue resolution and unavoidable intravascular eddy currents, which is undesirable for the observation of the vessel wall and lumen of the IVC. Additionally, CTA is unable to precisely assess the involvement of adjunctive small vessels such as the renal and hepatic veins because of insufficient blood flow caused by tumour obstruction. ${ }^{13}$ In this particular case, CTA had its limitations in the diagnosis of IVC LMS. The CT images thus gave rise to two misjudgements of great relevance for clinical management: (1) the right 

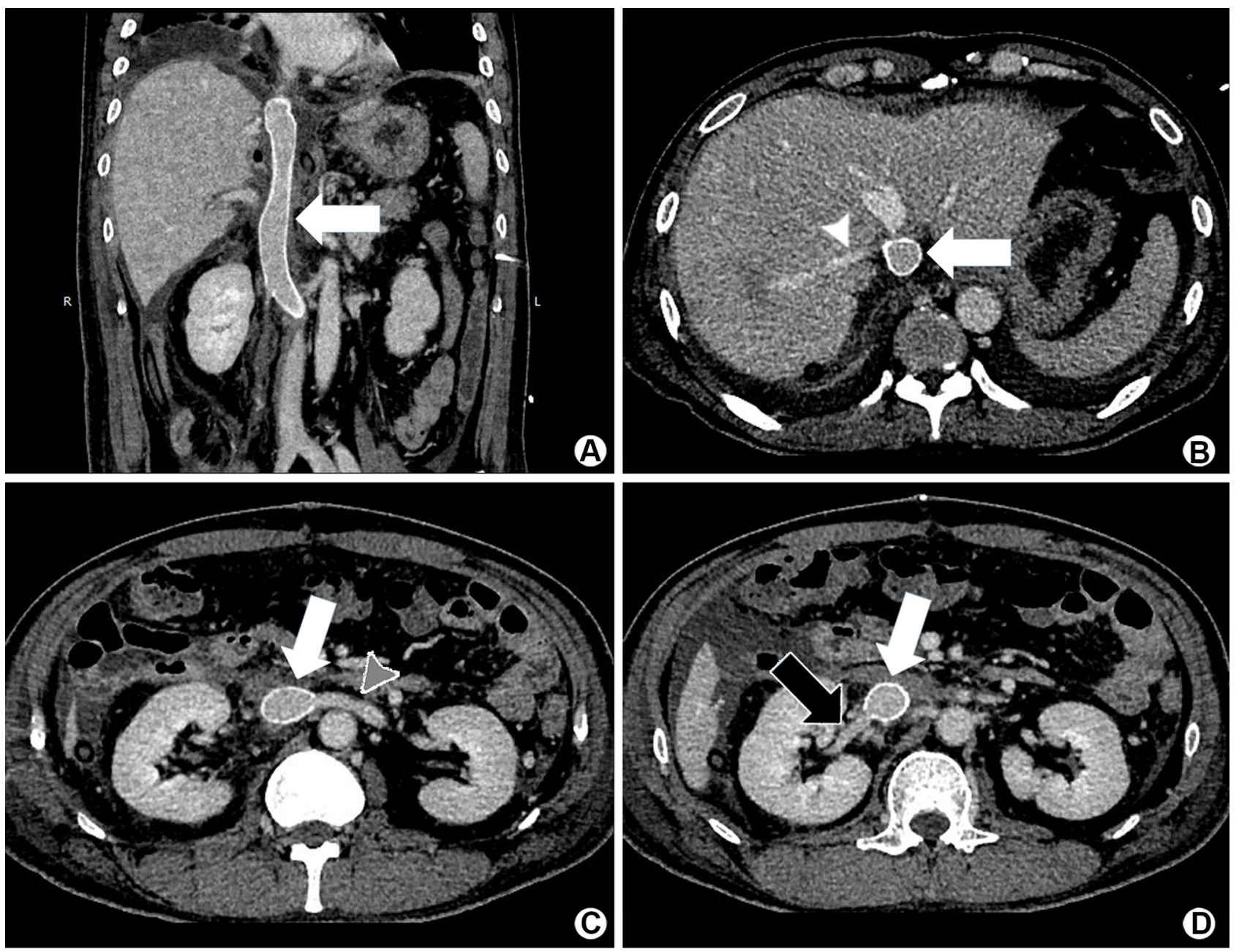

Figure 9 (A) Postoperative coronal enhanced venous phase CT image demonstrates patency of the artificial IVC from the entrance of right atrium to level of the renal vein (white arrow). (B-D) Axial enhanced venous phase CT images show the reserved right hepatic vein (white arrowhead), left renal vein (grey arrowhead) and right renal vein (black arrow). They are all well filled with contrast agent and perfectly connected to the artificial IVC (white arrow).

hepatic vein was unclear and was misdiagnosed as being invaded by the tumour; (2) the left renal vein was not well filled by contrast medium so it was also misdiagnosed as invaded by the tumour. Therefore, although CT is as sensitive as MRI to diagnose IVC leiomyosarcoma, it is not reliable for delineating the precise tumour extent and performing accurate preoperative evaluation. Finally, the ionising radiation and contrast induced adverse reactions due to CT make it an inferior method to MRI. ${ }^{21}$

However, to date, MRI has not received adequate attention, because CT examination is considered accurate enough and has been recommended repeatedly. ${ }^{7,22}$ However, some early studies were limited by the poor performance of ordinary sequences and low spatial resolution. Ganeshalingam et al suggested CT for the initial assessment of IVC LMS and MRI for the subsequent assessment of tumours in patients suitable for surgery. ${ }^{8}$ However, they also claimed that the use of gadolinium does not improve the evaluation or assessment of the extent of local invasion. To solve these problems, we combined several special sequences with high concentration contrast medium gadobutrol-enhanced MRI, significantly improving the diagnostic advantage. This can be partly explained by the fact that MRI has superior soft tissue resolution for early detection of tumour origin and invasion of local structures compared with $\mathrm{CT}$, even though diagnostic accuracy has not been explicitly compared between the two imaging techniques. MRI also allows improved characterisation of thrombus composition. In T2weighted imaging, leiomyosarcoma is usually iso- or hyperintense to muscle, whereas bland thrombus is typically isointense. $^{23}$ Additionally, high resolution T2-weighted black blood sequence is a special sequence that contributes 


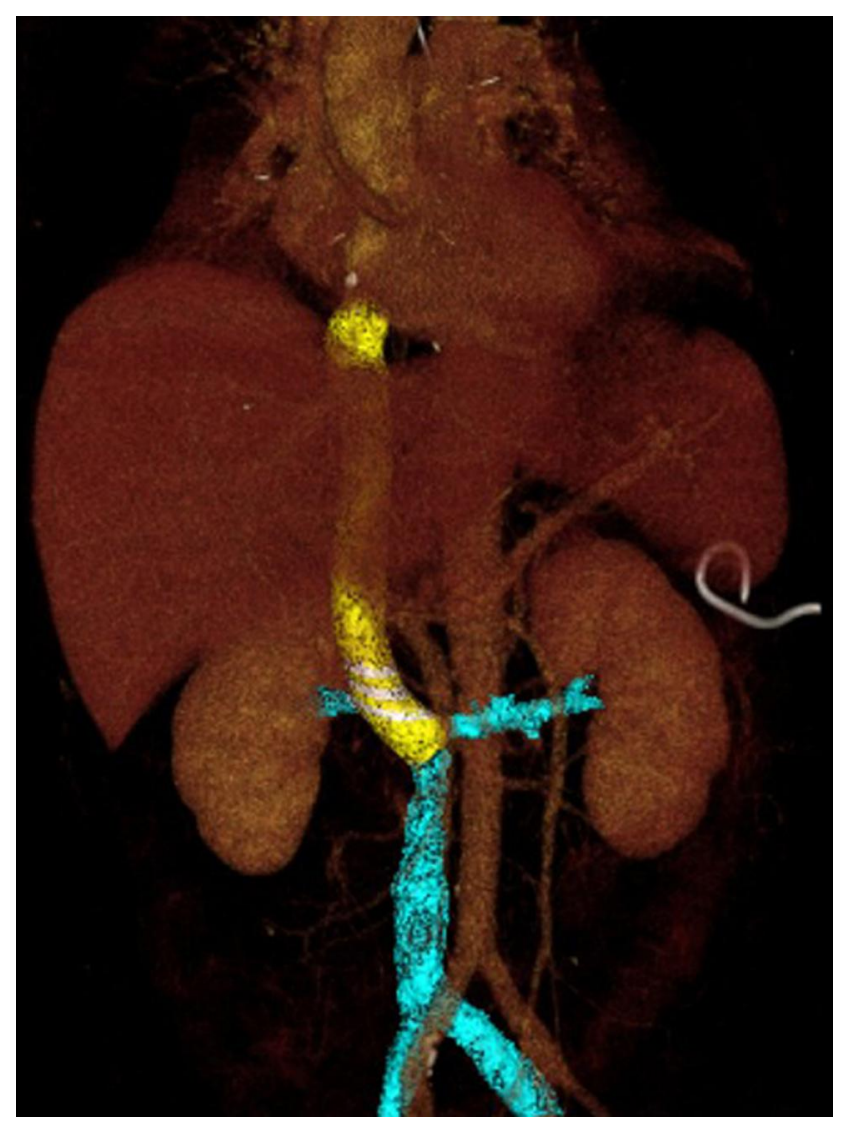

Figure 10 Postoperative CT 3D reconstruction shows the artificial IVC vessel coloured in yellow. The remaining lower segment of IVC and bilateral renal vein are coloured in blue.

to the observation of vessel walls. It differs from traditional methods such as magnetic resonance angiography (MRA) in that it emphasises the vessel wall rather than the lumen. Signal loss caused by saturation of slow flow and dephasing due to complex and turbulent flow is not visible in this sequence. ${ }^{24,25}$ Diffusion-weighted images of MRI can help identify thrombus characterisation. Due to increased cellular density and altered nuclear-to-cytoplasmic ratio, the lesions and tumour thrombus may display restricted diffusion and decreased apparent diffusion coefficients, resulting in hyperintensity on diffusion-weighted images and hypo-intensity in apparent diffusion coefficient map. Multiphase dynamic enhanced and delayed enhanced MRI are also more helpful in depicting blocked and slow-flowing vessels than CT, which usually has only two to three enhanced sequences. Most of all, we use gadobutrol (Gadovist, Bayer Healthcare Pharmaceuticals, Berlin, Germany), a type of high-concentration extracellular non-ionic macrocyclic gadolinium-based contrast medium displaying with high relativity and low osmolality and viscosity, to improve dynamic image enhancement. ${ }^{26,27}$ The physical properties of this gadolinium chelate allow for a concentration of 1 $\mathrm{mmol} \mathrm{Gd} / \mathrm{mL}$, which is double the concentration of conventional gadolinium contrast medium such as Gd-DTPA. The structure of gadobutrol confers a favourable safety profile not only in adults but also in children at standard doses. ${ }^{28,29}$ Gadobutrol has high relaxivity and provides the highest T1shortening effect per millilitre, ${ }^{30}$ resulting in an increase in signal intensity. ${ }^{31}$ Therefore, gadobutrol-enhanced MRI shows comparative imaging advantages, especially in MRA, which allows superior visualisation, more accurate vessel measurements, and a more definitive diagnosis. ${ }^{32}$ Several studies have reported that the use of gadobutrol can improve the delineation of small vessels with significantly increased signal-to-noise ratios and contrast-to-noise ratios in the vascular territory. ${ }^{33,34}$ Quantitative analysis of contrastenhanced MRA for large vessels also found that gadobutrol had a higher mean signal-to-noise ratio than that of gadolinium-DTPA. ${ }^{19}$ With this high concentration contrast agent, blood vessels can be better filled in, so that intravascular lesions and vessel walls can be better displayed. Altogether, these advantages explain why gadobutrol-enhanced MRI triumphed over CT in the accurate evaluation of IVC leiomyosarcoma.

\section{Conclusion}

To the best of our knowledge, this is the first time that high-resolution MRI angiography combined with high concentration gadobutrol has been used to precisely evaluate the preoperative diagnosis of primary leiomyosarcoma of the IVC. The results emphasise the important role of high-resolution MRI angiography examination with high-concentration contrast medium gadobutrol in the preoperative evaluation and diagnosis of this IVC LMS. The new imaging strategy provided vital information for the surgical operation and successful clinical management of the patient, suggesting that such an approach should be prioritised in the evaluation of venous lesions in future practice.

\section{Abbreviations}

IVC, inferior vena cava; LMS, leiomyosarcoma; MRA, magnetic resonance angiography; CTA, computed tomography angiography.

\section{Data Sharing Statement}

The data that support the findings of this study are not publicly available to protect patient privacy, but are available from the corresponding authors upon reasonable request. 


\section{Ethics Approval and Consent to Participate}

This research was approved by the The First Affiliated Hospital of Sun Yat-Sen University Institutional Ethics Committee. The patient provided written informed consent for the case details and accompanying images to be published.

\section{Authors' information}

Xiaoqi Zhou: Department of Radiology, The First Affiliated Hospital, Sun Yat-Sen University, zhouxq5@mail2.sysu.edu.cn

Meng Wang: Department of Radiology, The First Affiliated Hospital, Sun Yat-Sen University, wangm56@mail.sysu.edu.cn

Shaoqiang Li: Department of Hepatic Surgery, The First Affiliated Hospital, Sun Yat-Sen University, lisqsums@126.com

Huasong Cai: Department of Radiology, The First Affiliated Hospital, Sun Yat-Sen University, huimiezhiyan@163.com

Lijian Liang: Department of Hepatic Surgery, The First Affiliated Hospital, Sun Yat-Sen University, 13802951357@163.com

Zi-Ping Li: Department of Radiology, The First Affiliated Hospital, Sun Yat-Sen University, liziping163@163.com

Shi-Ting Feng: Department of Radiology, The First Affiliated Hospital, Sun Yat-Sen University, fengsht@mail.sysu.edu.cn

Zhenpeng Peng: Department of Radiology, The First Affiliated Hospital, Sun Yat-Sen University, 37595198@qq.com

Xuehua Li: Department of Radiology, The First Affiliated Hospital, Sun Yat-Sen University, lixuehua803@163.com.

\section{Author Contributions}

All authors made substantial contributions to conception and design, acquisition of data, or analysis and interpretation of data; took part in drafting the article or revising it critically for important intellectual content; gave final approval of the version to be published; and agree to be accountable for all aspects of the work. Xiaoqi Zhou and Meng Wang: Equal contributions to this work and are cofirst authors.

\section{Funding}

This work was funded by the National Natural Science Foundation of China (Grant numbers: 81971684, 81771908, 81600508, 81571750, and 81770654).

\section{Disclosure}

The authors report no conflicts of interest for this work.

\section{References}

1. Hollenbeck ST, Grobmyer SR, Kent KC, Brennan MF. Surgical treatment and outcomes of patients with primary inferior vena cava leiomyosarcoma. J Am Coll Surg. 2003;197:575-579. doi:10.1016/ S1072-7515(03)00433-2

2. Wachtel H, Jackson BM, Bartlett EK, et al. Resection of primary leiomyosarcoma of the inferior vena cava (IVC) with reconstruction: a case series and review of the literature. J Surg Oncol. 2015;111:328-333. doi:10.1002/jso.23798

3. Hilliard NJ, Heslin MJ, Castro CY. Leiomyosarcoma of the inferior vena cava: three case reports and review of the literature. Ann Diagn Pathol. 2005;9:259-266. doi:10.1016/j. anndiagpath.2005.05.001

4. Mingoli A, Cavallaro A, Sapienza P, Di Marzo L, Feldhaus RJ, Cavallari N. International registry of inferior vena cava leiomyosarcoma: analysis of a world series on 218 patients. Anticancer Res. 1996;16:3201-3205.

5. Mann GN, Mann LV, Levine EA, Shen P. Primary leiomyosarcoma of the inferior vena cava: a 2-institution analysis of outcomes. Surgery. 2012;151:261-267. doi:10.1016/j.surg.2010.10.011

6. Narata M, Okuhata Y, Abe K, et al. Primary leiomyosarcoma of the inferior vena cava: case report. Abdom Imaging. 2010;35:481-484. doi:10.1007/s00261-009-9549-0

7. Mu D, Wang D, Zhou K, Zhu B. Radiographic features of intraluminal leiomyosarcoma of the inferior vena cava: an atypical case report. Abdom Imaging. 2011;36:586-589. doi:10.1007/s00261-010-9673-x

8. Ganeshalingam S, Rajeswaran G, Jones RL, Thway K, Moskovic E. Leiomyosarcomas of the inferior vena cava: diagnostic features on cross-sectional imaging. Clin Radiol. 2011;66:50-56. doi:10.1016/j. crad.2010.08.004

9. Wachtel H, Gupta M, Bartlett EK, et al. Outcomes after resection of leiomyosarcomas of the inferior vena cava: a pooled data analysis of 377 cases. Surg Oncol. 2015;24:21-27. doi:10.1016/j. suronc.2014.10.007

10. Jiang H, Wang Y-X, Li B, et al. Surgical management of leiomyosarcoma of the inferior vena cava. Vascular. 2014;23:329-332. doi: $10.1177 / 1708538114547755$

11. Cananzi FC, Mussi C, Bordoni MG, et al. Role of surgery in the multimodal treatment of primary and recurrent leiomyosarcoma of the inferior vena cava. J Surg Oncol. 2016;114:44-49. doi:10.1002/ jso. 24244

12. Hemant D, Krantikumar R, Amita J, Chawla A, Ranjeet N. Primary leiomyosarcoma of inferior vena cava, a rare entity: imaging features. Australas Radiol. 2001;45:448-451. doi:10.1046/j.1440-1673.2001. 00955.x

13. Blum U, Wildanger G, Windfuhr M, Laubenberger J, Freudenberg N, Munzar T. Preoperative CT and MR imaging of inferior vena cava leiomyosarcoma. Eur J Radiol. 1995;20:23-27. doi:10.1016/0720048X(95)00608-S

14. Sessa B, Iannicelli E, Caterino S, et al. Imaging of leiomyosarcoma of the inferior vena cava: comparison of 2 cases and review of the literature. Cancer Imaging. 2010;10:80-84. doi:10.1102/1470-7330. 2010.0009 
15. Huang J, Liu Q, Lu JP, Wang F, Wang L, Jin AG. Primary intraluminal leiomyosarcoma of the inferior vena cava: value of MRI with contrast-enhanced MR venography in diagnosis and treatment. Abdom Imaging. 2011;36:337-341. doi:10.1007/s00261-010-9656-y

16. Chan G, Kroczak T, Drachenberg D. Leiomyosarcoma of the inferior vena cava with renal metastasis: an unusual case and diagnostic challenge. Canad Urolog Assoc J. 2014;8:358-360. doi:10.5489/ cuaj.2119

17. Liu L, Li X, Zhang Y. Hepatobiliary and pancreatic: leiomyosarcoma: unusual cause of inferior vena cava obstruction. $J$ Gastroenterol Hepatol. 2016;31:1384. doi:10.1111/jgh.13298

18. Bednarova I, Frellesen C, Roman A, Vogl TJ. Case 257: leiomyosarcoma of the inferior vena cava. Radiology. 2018;288:901-908. doi:10.1148/radiol.2018160821

19. Liu X, Zhang W, Li Z, et al. Improved display of abdominal contrast-enhanced MRA using gadobutrol: comparison with Gd-DTPA. Clin Radiol. 2019;74:978.e1-978.e7. doi:10.1016/j.crad. 2019.08.012

20. Fernandez HT, Kim PT, Anthony TL, Hamman BL, Goldstein RM, Testa G. Inferior vena cava reconstruction for leiomyosarcoma of Zone I-III requiring complete hepatectomy and bilateral nephrectomy with autotransplantation. $J$ Surg Oncol. 2015;112:481-485. doi: $10.1002 /$ jso. 24041

21. Liu Q, Lu JP, Wang F, et al. Detection of anomalous splenic artery aneurysms with three-dimensional contrast-enhanced MR angiography. Abdom Imaging. 2009;34:772-776. doi:10.1007/ s00261-008-9467-6

22. Wu H, Zhang H, Wang S, Wu X, Dong D, Liang C. Multimodality imaging of benign or malignant tumors in the vena cava and its main branches. Clin Imaging. 2017;43:50-59. doi:10.1016/j.clinimag.20 16.10 .014

23. Akin O, Dixit D, Schwartz L. Bland and tumor thrombi in abdominal malignancies: magnetic resonance imaging assessment in a large oncologic patient population. Abdom Imaging. 2011;36:62-68. doi:10.1007/s00261-010-9608-6

24. Edelman RR, Mattle HP, Wallner B, et al. Extracranial carotid arteries: evaluation with "black blood" MR angiography. Radiology. 1990;177:45-50. doi:10.1148/radiology.177.1.2399337

25. U-King-Im JM, Trivedi RA, Sala E, et al. Evaluation of carotid stenosis with axial high-resolution black-blood MR imaging. Eur Radiol. 2004;14:1154-1161. doi:10.1007/s00330-004-2245-0
26. Zizka J, Klzo L, Ferda J, Mrklovsky M, Bukac J. Dynamic and delayed contrast enhancement in upper abdominal MRI studies: comparison of gadoxetic acid and gadobutrol. Eur J Radiol. 2007;62:186-191. doi:10.1016/j.ejrad.2007.02.035

27. Scott LJ. Gadobutrol: a review of its use for contrast-enhanced magnetic resonance imaging in adults and children. Clin Drug Investig. 2013;33:303-314. doi:10.1007/s40261-013-0066-0

28. Hahn G, Sorge I, Gruhn B, et al. Pharmacokinetics and safety of gadobutrol-enhanced magnetic resonance imaging in pediatric patients. Invest Radiol. 2009;44:776-783. doi:10.1097/RLI.0b013e3181bfe2d2

29. Kunze C, Mentzel HJ, Krishnamurthy R, et al. Pharmacokinetics and safety of macrocyclic gadobutrol in children aged younger than 2 years including term newborns in comparison to older populations. Investig Radiol. 2016;51:50-57. doi:10.1097/RLI.0000000000000204

30. Shen Y, Goerner FL, Snyder C, et al. T1 relaxivities of gadolinium-based magnetic resonance contrast agents in human whole blood at 1.5, 3, and 7 T. Investig Radiol. 2015;50:330-338. doi:10.1097/RLI.0000000000000132

31. Port M, Corot C, Violas X, Robert P, Raynal I, Gagneur G. How to compare the efficiency of albumin-bound and nonalbumin-bound contrast agents in vivo: the concept of dynamic relaxivity. Investig Radiol. 2005;40:565-573. doi:10.1097/01.rli.0000175388.98721.9b

32. Fabrega-Foster KE, Agarwal S, Rastegar N, Haverstock D, Agris JM, Kamel IR. Efficacy and safety of gadobutrol-enhanced MRA of the renal arteries: results from GRAMS (Gadobutrol-enhanced renal artery MRA study), a prospective, intraindividual multicenter phase 3 blinded study. J Magnet Reson Imag. 2018;47:572-581. doi:10.1002/jmri.25774

33. Goyen M, Lauenstein TC, Herborn CU, Debatin JF, Bosk S, Ruehm SG. 0.5 M Gd chelate (Magnevist) versus 1.0 M Gd chelate (Gadovist): dose-independent effect on image quality of pelvic three-dimensional MR-angiography. J Magnet Reson Imag. 2001;14:602-607. doi:10.1002/jmri.1225

34. Herborn CU, Lauenstein TC, Ruehm SG, Bosk S, Debatin JF, Goyen M. Intraindividual comparison of gadopentetate dimeglumine, gadobenate dimeglumine, and gadobutrol for pelvic 3D magnetic resonance angiography. Investig Radiol. 2003;38:27-33. doi:10.10 97/00004424-200301000-00004
Cancer Management and Research

\section{Publish your work in this journal}

Cancer Management and Research is an international, peer-reviewed open access journal focusing on cancer research and the optimal use of preventative and integrated treatment interventions to achieve improved outcomes, enhanced survival and quality of life for the cancer patient.
The manuscript management system is completely online and includes a very quick and fair peer-review system, which is all easy to use. Visit http://www.dovepress.com/testimonials.php to read real quotes from published authors. 